\title{
Serological investigation of Leishmania infantum, Dirofilaria immitis and Angiostrongylus vasorum in dogs from southern Portugal
}

\author{
Carla Maia ${ }^{1,2,3^{*}}$, Mónica Coimbra ${ }^{4}$, Cláudia Ramos ${ }^{1}$, José Manuel Cristóvão ${ }^{1}$, Luís Cardoso ${ }^{5}$ and Lenea Campino ${ }^{1,6}$
}

\begin{abstract}
Background: Leishmaniosis, dirofilariosis and angiostrongylosis are parasitic diseases of established importance in dogs worldwide. The aim of the present report was to determine all together levels of infection with or exposure to Leishmania infantum, Dirofilaria immitis and Angiostrongylus vasorum in dogs from the Algarve region, in southern Portugal.

Findings: Serum samples were obtained from a total of 170 apparently healthy dogs. Antibodies to $L$. infantum were detected by the direct agglutination test; and antigens of D. immitis and of $A$. vasorum by an enzyme-linked immunosorbent assay and an immunochromatography test, respectively. Antibodies to $L$. infantum were found in $18.2 \%(31 / 170)$ of dogs, while D. immitis antigen was detected in 9.4\% (16/170). None of 120 dogs tested out of the 170 under investigation had evidence of infection with A. vasorum. D. immitis infection was more prevalent in dogs aged 84-204 months than in those younger than 12 months. Three dogs were positive to both $L$. infantum and D. immitis.
\end{abstract}

Conclusions: Dogs living in southern Portugal are at risk of leishmaniosis and dirofilariosis. This scenario should make the veterinary community, local dog owners and also tourists from non-endemic countries coming on vacation with their pets aware of the need of effective prophylactic measures, in order to protect animals and public health.

Keywords: Leishmania infantum, Dirofilaria immitis, Angiostrongylus vasorum, Dog, Portugal, Seroprevalence

\section{Findings}

Leishmaniosis, dirofilariosis and angiostrongylosis are parasitic diseases of established importance in dogs worldwide. Leishmaniosis caused by the protozoan Leishmania infantum (Trypanosomatida, Trypanosomatidae) and heartworm disease caused by the nematode Dirofilaria immitis (Spirurida, Onchocercidae) are vector-borne zoonoses with importance in veterinary medicine and widely present in the Mediterranean [1-3]. Both parasitoses are endemic in Portugal, with an overall national seroprevalence of $6.3 \%$ [4] and 3.6-8.9\% [5], respectively.

\footnotetext{
* Correspondence: carlamaia@ihmt.unl.pt

${ }^{1}$ Unidade de Parasitologia Médica, Instituto de Higiene e Medicina Tropical (IHMT), Universidade Nova de Lisboa (UNL), Rua da Junqueira 100, 1349-008 Lisboa, Portugal

${ }^{2}$ Global Health and Tropical Medicine, IHMT-UNL, Rua da Junqueira 100,

1349-008 Lisboa, Portugal

Full list of author information is available at the end of the article
}

Canine leishmaniosis is a systemic chronic condition whose clinical manifestations usually include lymphadenomegaly, cutaneous alterations, loss of body weight, ocular signs, epistaxis, onychogryphosis and lameness [6]. Canine cardiopulmonary dirofilariosis is associated with a dry chronic cough, exercise intolerance, dyspnoea, weakness, weight loss, epistaxis, cyanosis and even congestive heart failure [7].

Canine angiostrongylosis, caused by the nematode Angiostrongylus vasorum (Strongylida, Angiostrongylidae), is an emerging disease with an increasing number of cases diagnosed in the last few years, and with a high established relevance in several regions of Europe [8]. The disease is associated with an array of clinical problems, including cardiorespiratory, coagulopathic and neurologic signs [8]. Albeit the laboratory Baermann method, using faeces, is the gold-standard to detect first-stage larvae (L1), serological techniques for the detection of circulating $A$. 
vasorum antigens or specific antibodies have recently been developed $[9,10]$. In Europe prevalence as assessed by the detection of circulating parasite antigens in dogs has been found to range from $0.5 \%$ in Germany to $1.3 \%$ in the United Kingdom, while the detection of specific antibodies ranged from $1.3 \%$ in Poland to $3.2 \%$ in the UK $[11,12]$. In coastal areas of south-central Portugal, $1.8 \%$ (6/341) of shelter dogs were antigen-positive, while antibodies against the parasite were detected in $2.4 \%$ of those dogs [13].

The present report aimed at determining the levels of infection with or exposure to vector-borne parasites ( $L$. infantum and D. immitis) and parasites acquired by ingestion of intermediate hosts (A. vasorum) in dogs from the Algarve region, the southernmost part of continental Portugal, in view of the scarcity of epidemiological studies targeting these parasites all together in canine populations.

From November 2011 to May 2014, a total of 170 apparently healthy dogs from the Algarve were studied. Domestic dogs were randomly included after owners' informed consent. In the case of stray dogs, a written consent for enrolment was obtained from the legal detainer, i.e. the person in charge of the rescue association. Whenever available, data on gender, breed, age, fur length, life style, living conditions, prophylactic use of sand fly repellents and of macrocyclic lactones were registered for each dog (Tables 1 and 2). Blood samples (2-3 ml) were collected by cephalic or jugular venipuncture. Serum was separated by centrifugation and stored at $-20^{\circ} \mathrm{C}$ until use.

This study was approved by the ethics board of the Institute of Hygiene and Tropical Medicine, Universidade Nova de Lisboa (IHMT-UNL) as complying with the Portuguese legislation for the protection of animals (Law no. 92/1995).

The direct agglutination test (DAT) for titration of specific antibodies was performed in 170 dogs using a standard freeze-dried Leishmania antigen as previously described [14]. The DAT cut-off was established at a titre of 400 [15]. D. immitis antigen was detected in 170 animals by using a commercial enzyme-linked immunosorbent assay kit (PetChek Canine Heartworm Antigen Test $^{\ominus}$, IDEXX Laboratories, Westbrook, ME, USA). Detection of $A$. vasorum antigens was performed in only 120 dogs out of the 170 under investigation by using a commercial immunochromatography test (Angio Detect ${ }^{\mathrm{mm}}$ Test, IDEXX Laboratories).

The exact binomial test established confidence intervals (CI) with a $95 \%$ confidence level. The chi-square or Fisher's exact tests were used to compare percentages of positivity among categories of the same independent variables and also the total prevalence of each agent [16]. A $p$ value $<0.05$ was considered as statistically significant. Analyses were performed with StatLib and SPSS $^{\circ} 21$ software for Windows.

\begin{tabular}{|c|c|c|c|}
\hline $\begin{array}{l}\text { Independent } \\
\text { variable/category }\end{array}$ & $\begin{array}{l}\text { No. }(\%) \text { of } \\
\text { dogs tested }\end{array}$ & $\%$ of positive & $95 \% \mathrm{Cl}$ \\
\hline Gender & 169 & $p=0.161$ & \\
\hline Female & $79(46.7)$ & 22.8 & $7.1-22.1$ \\
\hline Male & $90(53.3)$ & 13.3 & $14.1-33.6$ \\
\hline Breed & 167 & $p=0.747$ & \\
\hline Defined & $93(55.7)$ & 19.4 & $8.7-26.6$ \\
\hline Mongrel & $74(44.3)$ & 16.2 & $11.9-28.8$ \\
\hline Fur length & 81 & $p=0.393$ & \\
\hline Short & $47(58.0)$ & 10.6 & $3.5-23.1$ \\
\hline Medium or long & $34(42.0)$ & 2.9 & $0.1-15.3$ \\
\hline Age (months) & 155 & $p=0.530$ & \\
\hline$[2-11]$ & $31(20.0)$ & 16.1 & $5.5-33.7$ \\
\hline [12-83] & $74(47.7)$ & 21.6 & $12.9-32.7$ \\
\hline [84-204] & $50(32.3)$ & 14.0 & $5.8-26.7$ \\
\hline Lifestyle & 170 & $p=1.0$ & \\
\hline Domestic & $157(92.4)$ & 18.5 & $12.7-25.4$ \\
\hline Stray & $13(7.6)$ & 15.4 & $1.9-45.4$ \\
\hline Housing & 161 & $p=0.280$ & \\
\hline $\begin{array}{l}\text { Exclusively or mainly } \\
\text { indoors }\end{array}$ & $69(42.9)$ & 23.2 & $13.9-34.9$ \\
\hline $\begin{array}{l}\text { Mainly or exclusively } \\
\text { outdoors }\end{array}$ & $92(57.1)$ & 15.2 & $8.6-24.2$ \\
\hline Sand fly repellents* & 145 & $p=1.0$ & \\
\hline Yes & $58(46.9)$ & 17.6 & $9.5-28.8$ \\
\hline No & $77(53.1)$ & 16.9 & $9.3-27.1$ \\
\hline D. immitis & 170 & $p=1.0$ & \\
\hline Negative & $154(90.6)$ & 18.2 & $12.4-25.2$ \\
\hline Positive & $16(9.4)$ & 18.8 & $4.0-45.6$ \\
\hline Total & 170 & 18.2 & $12.7-24.7$ \\
\hline
\end{tabular}

*Deltamethrin and/or imidacloprid and permethrin.

Antibodies to L. infantum were found in $31(18.2 \%)$ dogs, at the dilutions of 1:400 ( $\mathrm{n}=5), 1: 800(\mathrm{n}=2), 1: 1,600$ $(\mathrm{n}=2), 1: 3,200(\mathrm{n}=5), 1: 6,400 \quad(\mathrm{n}=6), 1: 12,800(\mathrm{n}=6)$, 1:25,600 $(\mathrm{n}=4)$ and 1:51,200 $(\mathrm{n}=1)$ (Table 1). The seroprevalence of Leishmania found in the present study was higher than the ones obtained in randomly screened animals (4.7\%) [4] and in clinically healthy dogs (3.8\%) [5] from the Algarve region that were presented to veterinary clinics, suggesting an apparent increase in the level of Leishmania infection in the Algarve [17].

D. immitis antigen was detected in $16(9.6 \%)$ animals (Table 2). This prevalence is apparently higher than the values recently obtained in three coastal areas of southcentral Portugal (7.9\%) [13] and in other areas further 
Table 2 Prevalence of Dirofilaria immitis antigen in dogs from southern Portugal according to independent variables and their categories

\begin{tabular}{|c|c|c|c|}
\hline $\begin{array}{l}\text { Independent } \\
\text { variable/category }\end{array}$ & $\begin{array}{l}\text { No. (\%) of } \\
\text { dogs tested }\end{array}$ & $\%$ of positive & $95 \% \mathrm{Cl}$ \\
\hline Gender & 169 & $p=0.297$ & \\
\hline Female & 79 (46.7) & 6.3 & $2.1-14.2$ \\
\hline Male & $90(53.3)$ & 12.2 & $6.3-20.8$ \\
\hline Breed & 167 & $p=0.071$ & \\
\hline Defined & $93(55.7)$ & 5.4 & $1.8-12.1$ \\
\hline Mongrel & $74(44.3)$ & 14.9 & $7.7-25.0$ \\
\hline Fur length & 81 & $p=1.0$ & \\
\hline Short & $47(58.0)$ & 6.4 & $1.3-17.5$ \\
\hline Medium or long & $34(42.0)$ & 5.9 & $0.7-19.7$ \\
\hline Age (months) & 155 & $p=\mathrm{ND}$ & \\
\hline$[2-11]$ & $31(20.0)$ & $0.0^{\mathrm{a}}$ & $0.0-11.2$ \\
\hline [12-83] & $74(47.7)$ & 9.5 & $3.9-18.5$ \\
\hline [84-204] & $50(32.3)$ & $16.0^{\mathrm{a}}$ & $7.2-29.1$ \\
\hline Lifestyle & 170 & $p=0.352$ & \\
\hline Domestic & $157(92.4)$ & 8.9 & $5.0-14.5$ \\
\hline Stray & $13(7.6)$ & 15.4 & $1.9-45.4$ \\
\hline Housing & 161 & $p=1.0$ & \\
\hline $\begin{array}{l}\text { Exclusively or mainly } \\
\text { indoors }\end{array}$ & $69(42.9)$ & 8.7 & $3.3-18.0$ \\
\hline $\begin{array}{l}\text { Mainly or exclusively } \\
\text { outdoors }\end{array}$ & $92(57.1)$ & 9.8 & $4.6-17.8$ \\
\hline Macrocyclic lactones* & 143 & $p=0.128$ & \\
\hline Yes & $23(16.1)$ & 0.0 & $0.0-14.8$ \\
\hline No & $120(83.9)$ & 10.8 & $5.9-17.8$ \\
\hline Leishmania spp. & 170 & $p=1.0$ & \\
\hline Negative & $139(81.8)$ & 9.4 & $5.1-15.4$ \\
\hline Positive & $31(18.2)$ & 9.7 & $2.0-25.7$ \\
\hline Total & 170 & 9.4 & $5.5-14.8$ \\
\hline
\end{tabular}

*Ivermectin and/or milbemycin oxime or moxydectin; ${ }^{a} p=0.021$.

north (2.1\%) [18], suggesting that the climatic conditions in the southern regions of the country might be more favourable to the proliferation and abundance of vectors of $D$. immitis $[19,20]$. An exception to this trend was reported in Figueira da Foz, located in the coastal edge of the central region of Portugal, where $25.7 \%$ of the dogs were found infected [21].

Prevalence of $D$. immitis infection was significantly higher in dogs aged 84-204 months than in dogs younger than 12 months $(p=0.021)$, which may be explained by a cumulative exposure of older animals to the parasite through an increased contact with the vectors [5,21]. Although the difference was not statistically significant $(p=0.128), D$. immitis antigens were not detected in any of the animals receiving macrocyclic lactones; anyhow, this finding suggests the importance of using prophylaxis in areas where dirofilariosis is endemic.

Cross-reactivity between $D$. immitis and A. vasorum in dogs experimentally infected with the latter has previously been reported for some heartworm antigen test kits [22]. However, in the present study no cross-reaction between both nematodes seem to have happened, as $A$. vasorum antigens were not detected in any of the dogs tested (0.0\%; 95\% CI: 0.0-3.0). The non-detection of this parasite, in comparison with the survey $(1.8 \%)$ carried out in other coastal areas of Portugal [14], might be related with the sensitivity of the present test (i.e. 98.1\% [23]), with seasonal factors and/or with the surveyed canine populations, as in the previous work all animals were shelter dogs, probably at a higher risk of contact with the intermediate host (i.e. snails and slugs), while in the present study most of the dogs were pets. Nevertheless, larger surveys and using different techniques (Baerman method, detection of specific antibodies and antigens) are necessary to gain more information on the diffusion of $A$. vasorum, for which the actual national distribution is poorly acquainted.

In the present study, no statistical association was found between positivities to L. infantum and D. immitis. Only three dogs were positive to both $L$. infantum and $D$. immitis. Co-infections with these two vectorborne pathogens are relatively frequent in dogs living in geographic areas where the presence of competent vectors for the different pathogens overlap [5,24]. Although in this study the three animals positive to both parasites were apparently healthy, it is important to keep in mind that the occurrence of co-infections with vector-borne pathogens might induce uncharacteristic clinical outcomes that will further complicate diagnosis, treatment and prognosis [25].

The findings of this study reveal that dogs from southern Portugal are at risk of acquiring two of the three parasites assessed (i.e. the agents of leishmaniosis and dirofilariosis). They also reinforce the importance to alert the veterinary community, local dog owners as well as tourists from nonendemic countries coming on vacation with their pets to the need of prophylactic measures, such as insect repellents and macrocyclic lactones, in order to defend animals and public health.

\section{Competing interests}

The authors declare that they have no competing interests.

\section{Authors' contributions}

CM planned, designed and supervised the study, and wrote the manuscript; MC collected samples and clinical data; CR and JMC performed serological analyses; LuC performed data analysis and revised the manuscript; LeC reviewed the manuscript. All authors read and approved the final manuscript. 


\section{Acknowledgements}

This work was supported by Centro de Malária e outras Doenças Tropicais, IHMT-UNL, Portugal, and EU grant FP7-261504 EDENext, and is catalogued by the EDENext Steering Committee as EDENext 294 (http://www.edenext. eu). The contents of this publication are the sole responsibility of the authors and do not necessarily reflect the views of the European Commission. The authors thank the cooperation of veterinarians, auxiliary staff, dog owners and shelters that contributed with or allowed collection of samples, and also acknowledge Servive Portugal (Valença, Portugal) for their assistance with the acquisition of Angio Detect ${ }^{\mathrm{TM}}$ Test. C. Maia (SFRH/BPD/44082/2008) holds a scholarship from Fundação para a Ciência e a Tecnologia, Ministério da Educação e Ciência, Portugal. The work of C. Maia and L. Cardoso was done under the frame of EurNegVec COST Action TD1303.

Publication of this paper has been sponsored by Bayer HealthCare - Animal Health Division in the framework of the 10th CVBD World Forum Symposium.

\section{Author details}

'Unidade de Parasitologia Médica, Instituto de Higiene e Medicina Tropical (IHMT), Universidade Nova de Lisboa (UNL), Rua da Junqueira 100, 1349-008 Lisboa, Portugal. ${ }^{2}$ Global Health and Tropical Medicine, IHMT-UNL, Rua da Junqueira 100, 1349-008 Lisboa, Portugal. ${ }^{3}$ Faculdade de Medicina Veterinária, Universidade Lusófona de Humanidades e Tecnologias (ULHT), Lisboa, Portugal. ${ }^{4}$ Clínica Veterinária Porto Seguro, Rua Patrão Joaquim Casaca, Edifício Majuca, 8700-507 Olhão, Portugal. ${ }^{5}$ Departamento de Ciências Veterinárias, Escola de Ciências Agrárias e Veterinárias, Universidade de Trás-os-Montes e Alto Douro (UTAD), Quinta de Prados, 5000-801 Vila Real, Portugal. ${ }^{6}$ Departamento de Ciências Biomédicas e Medicina, Universidade do Algarve, Campus de Gambelas, 8005-139 Faro, Portugal.

Received: 3 December 2014 Accepted: 25 February 2015

Published online: 23 March 2015

\section{References}

1. Capelli G, Frangipane di Regalbono A, Simonato G, Cassini R, Cazzin S, Cancrini G, et al. Risk of canine and human exposure to Dirofilaria immitis infected mosquitoes in endemic areas of Italy. Parasit Vectors. 2013;6:60.

2. Miró G, Montoya A, Roura X, Gálvez R, Sainz A. Seropositivity rates for agents of canine vector-borne diseases in Spain: a multicentre study. Parasit Vectors. 2013;6:117.

3. Genchi C, Bowman D, Drake J. Canine heartworm disease (Dirofilaria immitis) in Western Europe: survey of veterinary awareness and perceptions. Parasit Vectors. 2014;7:206.

4. Cortes S, Vaz Y, Neves R, Maia C, Cardoso L, Campino L. Risk factors for canine leishmaniasis in an endemic Mediterranean region. Vet Parasitol. 2012;189:189-96.

5. Cardoso L, Mendão C, Carvalho LM. Prevalence of Dirofilaria immitis, Ehrlichia canis, Borrelia burgdorferi sensu lato, Anaplasma spp. and Leishmania infantum in apparently healthy and CVBD-suspect dogs in Portugal - a national serological study. Parasit Vectors. 2012;5:62.

6. Campino L, Maia C. The role of reservoirs: canine leishmaniasis. In: Ponte-Sucre A, Padron-Nieves M, Diaz E, editors. Drug resistance in Leishmania parasites - consequences, molecular mechanism and possible treatments. Vienna: Springer Verlag; 2012. p. 45-64.

7. McCall JW, Genchi C, Kramer LH, Guerrero J, Venco L. Heartworm disease in animals and humans. Adv Parasitol. 2008;66:193-285.

8. Helm JR, Morgan ER, Jackson MW, Wotton P, Bell R. Canine angiostrongylosis: an emerging disease in Europe. J Vet Emerg Crit Care. 2010;20:98-109.

9. Schnyder M, Tanner I, Webster P, Barutzki D, Deplazes P. An ELISA for sensitive and specific detection of circulating antigen of Angiostrongylus vasorum in serum samples of naturally and experimentally infected dogs. Vet Parasitol. 2011;179:152-8.

10. Schnyder M, Stebler K, Naucke TJ, Lorentz S, Deplazes P. Evaluation of a rapid device for serological in-clinic diagnosis of canine angiostrongylosis. Parasit Vectors. 2014;7:72.

11. Schnyder M, Schaper R, Bilbrough G, Morgan ER, Deplazes P. Seroepidemiological survey for canine angiostrongylosis in dogs from Germany and the UK using combined detection of Angiostrongylus vasorum antigen and specific antibodies. Parasitology. 2013;140:1442-50.
12. Schnyder M, Schaper R, Pantchev N, Kowalska D, Szwedko A, Deplazes P. Serological detection of circulating Angiostrongy/us vasorum antigen- and parasite-specific antibodies in dogs from Poland. Parasitol Res. 2013;112:109-17.

13. Alho AM, Schnyder M, Meireles J, Belo S, Deplazes P, Carvalho LM. Preliminary results on the seroprevalence of Angiostrongylus vasorum and co-infection with Dirofilaria immitis in shelter dogs from Portugal [abstract] Parasit Vectors. 2014;7 Suppl 1:026.

14. Schallig HD, Cardoso L, Semião-Santos SJ. Seroepidemiology of canine leishmaniosis in Évora (southern Portugal): 20-year trends. Parasit Vectors. 2013;6:100.

15. Maia C, Nunes M, Cristóvão J, Campino L. Experimental canine leishmaniasis: clinical, parasitological and serological follow-up. Acta Trop. 2010;116:193-9.

16. Petrie A, Watson P. Statistics for veterinary and animal science. 2 nd ed. Oxford: Blackwell Publishing; 2006.

17. Maia C, Dionísio L, Afonso MO, Neto L, Cristóvão JM, Campino L. Leishmania infection and host-blood feeding preferences of phlebotomine sandflies and canine leishmaniasis in an endemic European area, the Algarve Region in Portugal. Mem Inst Oswaldo Cruz. 2013;108:481-7.

18. Vieira L, Silvestre-Ferreira AC, Fontes-Sousa AP, Balreira AC, Morchón R,

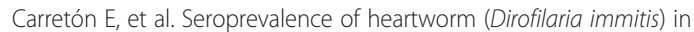
feline and canine hosts from central and northern Portugal. J Helminthol. 2014;14:1-5.

19. Alho AM, Nunes T, Rinaldi L, Meireles J, Belo S, Deplazes P, et al. Transmission risk of dirofilariosis in Portugal [abstract]. Parasit Vectors. 2014;7(Suppl1):016.

20. Roiz D, Ruiz S, Soriguer R, Figuerola J. Climatic effects on mosquito abundance in Mediterranean wetlands. Parasit Vectors. 2014;7:333.

21. Vieira AL, Vieira MJ, Oliveira JM, Simões AR, Diez-Baños P, Gestal J. Prevalence of canine heartworm (Dirofilaria immitis) disease in dogs of central Portugal. Parasite. 2014;21:5.

22. Schnyder M, Deplazes P. Cross-reactions of sera from dogs infected with Angiostrongylus vasorum in commercially available Dirofilaria immitis test kits. Parasit Vectors. 2012;5:258.

23. IDEXX. Angio Detect ${ }^{\mathrm{TM}}$ Test - the only in-clinic antigen detection test for Angiostrongylus vasorum. Westbrook: IDEXX Laboratories, 2015.

24. Tabar MD, Altet L, Martínez V, Roura X. Wolbachia, filariae and Leishmania coinfection in dogs from a Mediterranean area. J Small Anim Pract. 2013:54:174-8.

25. De Tommasi AS, Otranto D, Dantas-Torres F, Capelli G, Breitschwerdt EB, de Caprariis D. Are vector-borne pathogen co-infections complicating the clinical presentation in dogs? Parasit Vectors. 2013;6:97.

\section{Submit your next manuscript to BioMed Central and take full advantage of:}

- Convenient online submission

- Thorough peer review

- No space constraints or color figure charges

- Immediate publication on acceptance

- Inclusion in PubMed, CAS, Scopus and Google Scholar

- Research which is freely available for redistribution

Submit your manuscript at www.biomedcentral.com/submit
C) Biomed Central 